Tohoku J. Exp. Med., 2011, 224, 41-46

\title{
Tudor Domain-Containing Protein 4 as a Potential Cancer/Testis Antigen in Liver Cancer
}

\author{
Heejei Yoon, ${ }^{1}$ Hanna Lee, ${ }^{1}$ Hee Jin Kim, ${ }^{1, *}$ Kwon Tae You, ${ }^{1, \dagger}$ Young Nyun Park, ${ }^{1}$ \\ Hoguen Kim ${ }^{1}$ and Hyunki Kim ${ }^{1}$
}

${ }^{1}$ Department of Pathology and BK21 Project for Medical Science, Yonsei University College of Medicine, Seoul, Republic of Korea

The poor prognosis of liver cancer demands the development of new diagnostic markers and therapeutic strategies. Cancer/testis (CT) antigens are expressed in the testis and cancerous tissues, but not in adult somatic cells. Given their tumor-specific expression, CT antigens are potential molecular markers for tumor diagnosis and targets for cancer immunotherapy. To identify novel CT antigens for liver cancer, we examined mRNA expression of hitherto unknown CT antigen candidates, tudor domain-containing protein (TDRD) 1, 4 and 5 in three types of liver cancer; hepatocellular carcinoma $(\mathrm{HCC}, n=28)$, cholangiocarcinoma (CC, $n=5)$ and combined HCC-CC $(n=8)$, with matched non-tumorous liver tissues. The TDRD1, 4 and 5 are known as being specifically expressed in the testis. TDRD1 and 5 are essential for male germ cell development. On RT-PCR analysis, TDRD1 mRNA was expressed in both HCCs and non-tumorous liver tissues, and TDRD5 mRNA was expressed in normal colonic and gastric mucosal tissues. Thus, TDRD1 and TDRD5 are not candidates for CT antigens. TDRD4 mRNA was expressed in the testis but not in other normal tissues, including colonic mucosa, gastric mucosa, and liver tissues. TDRD4 mRNA was expressed in 7 of the 41 liver cancers: $4 \mathrm{HCCs}, 1 \mathrm{CC}$ and 2 combined HCC-CCs. The TDRD4 mRNA expression was not significantly associated with patient age, tumor size, pathologic stages, hepatitis B virus infection, or CD133 expression. In conclusion, TDRD4 mRNA is expressed in a subset of liver cancers, and TDRD4 is a candidate CT antigen for liver cancer.

Keywords: tudor domain-containing protein; cancer/testis antigen; hepatocellular carcinoma; cholangiocarcinoma; male germ cells

Tohoku J. Exp. Med., 2011, 224 (1), 41-46. C 2011 Tohoku University Medical Press

Despite many efforts over the last decade to overcome liver cancer, the 5-year survival rate for patients with liver cancer remains less than $10 \%$ in the United States. This is partially due to an advanced disease stage at the time of initial diagnosis and frequent recurrence of liver cancer after standard therapy (Farazi and DePinho 2006). Hepatocellular carcinoma (HCC) is the most common primary liver cancer in the adult, which accounts for more than $75 \%$ of cases. Cholangiocarcinoma (CC) is the second most common primary liver cancers, which accounts for about $10 \%$ of cases (Theise et al. 2010). Combined hepatocellular-cholangiocarcinoma (combined HCC-CC) that likely originates from both hepatocyte and cholangiocyte progenitor cells comprises less than $1 \%$ of primary liver cancers and shows unfavorable outcomes compared to HCC (Koh et al. 2005; Wang et al. 2010). The low survival rate of liver cancer highlights the need for the development of new diagnostic and therapeutic strategies.

Cancer/testis (CT) antigens are normally expressed by germ cells and trophoblasts, and are aberrantly expressed in a range of human cancers (Simpson et al. 2005; Parmigiani et al. 2006). Because certain CT antigens elicit high humoral and cellular immune responses in cancer patients, they are considered attractive targets for cancer immunotherapy (Costa et al. 2007; Caballero and Chen 2009). Furthermore, it has been suggested that these CT antigens may serve as markers for cancer stem cells (Simpson et al. 2005; Costa et al. 2007), which are often isolated using the stem cell marker CD133 from solid tumors, including HCC (Wu and $\mathrm{Wu}$ 2009). More than $40 \mathrm{CT}$ antigen families have been identified, including melanoma antigen family, B melanoma antigen family, and New York esophageal squa-

Received December 24, 2010; revision accepted for publication April 7, 2011. doi: 10.1620/tjem.224.41

Correspondence: Hyunki Kim, M.D., Ph.D., Department of Pathology, Yonsei University College of Medicine, 134 Sinchon-dong, Seodaemun-gu, 120-752, Seoul, Republic of Korea.

e-mail: kimhyunki@yuhs.ac

*Present address: Department of Molecular Microbiology and Immunology, University of Southern California, Los Angeles, CA 90033, USA.

†Present address: School of Biological Sciences, Seoul National University, Seoul, Republic of Korea. 
mous cell carcinoma 1 family (Simpson et al. 2005; Hofmann et al. 2008; Almeida et al. 2009).

In an attempt to find novel $\mathrm{CT}$ antigens for liver cancer, we investigated the tumor-specific mRNA expression of tudor domain-containing protein (TDRD) 1, 4 and 5 in liver cancers. TDRD1, 4 and 5 have been reported as testisspecific proteins (Wang et al. 2001; Smith et al. 2004). TDRD1 contains four tudor domains and a zinc-finger domain. Tdrd1 is essential for male germ-cell differentiation and germinal granule formation which have been assumed to serve as storage sites for proteins and RNAs during male germ-cell differentiation in mice (Chuma et al. 2003, 2006). TDRD1 was reported to be a CT antigen expressed in several cancers, including prostate cancer, breast carcinoma and bladder carcinoma (Loriot et al. 2003).

TDRD4 (also called ring finger protein 17) is also essential for male germ-cell differentiation and germinal granule formation in mice (Pan et al. 2005). TDRD4 contains a RING (Really Interesting New Gene) finger motif and tudor domains, and interacts with Mad (Max dimerization) proteins (Yin et al. 1999). Mad proteins repress the transcription of Myc-responsive genes by competing with Myc for binding to the Max transcription factor (Gallant 2006). TDRD4 promotes transactivation of the c-Myc oncogene by sequestering Mad proteins in the cytoplasm (Yin et al. 2001). Tdrd5 expression is restricted to the testis during mouse embryonic development and is co-expressed with the stem cell marker Oct-4 in mouse testis (Smith et al. 2004).

In this study, we asked whether TDRD1, 4 and 5 are $\mathrm{CT}$ antigens and if their expression is associated with the expression of stem cell markers, cytokeratin 19 (CK19) and CD133 in three types of liver cancer: 28 HCCs, five CCs and eight combined HCC-CCs. We also evaluated the relationship between expression of TDRD 4 and 5, and clinicopathologic factors of the HCCs, including a state of hepatitis $\mathrm{B}$ virus infection.

\section{Materials and Methods}

Specimens and cell lines

A total of $28 \mathrm{HCC}$ tissues and matched adjacent normal liver tissues were included in this study. Eight combined HCC-CCs and five CCs were also included for comparison. This study was approved by the Institutional Review Board of Yonsei University, and informed consent was received from all patients. The data of pathological features of the specimens were obtained from medical records. Samples were obtained from the Tissue Bank which is supported by the Liver Cancer Specimen Bank of the National Research Resource Bank Program funded by the Korea Science and Engineering Foundation of the Ministry of Science and Technology. The tumor tissue and adjacent non-diseased tissue were snap frozen in liquid nitrogen immediately following dissection and stored at $-80^{\circ} \mathrm{C}$ until use. A cryostat was used to remove non-diseased tissue and infiltrating lymphocytes from the tumor tissue in order to increase the tumor volume by at least $70 \%$.

Liver cancer cell lines, Hep3B, were grown in RPMI 1640 sup- plemented with $10 \%$ fetal bovine serum and $100 \mathrm{U} / \mathrm{ml}$ penicillin and $100 \mu \mathrm{g} / \mathrm{ml}$ streptomycin (Hyclone, Logan, UT, USA). Total RNA from normal colonic mucosa, normal stomach mucosa, normal liver and fetal liver was purchased from Stratagene (La Jolla, CA, USA).

\section{Semiquantitative RT-PCR and real-time PCR}

Total RNA was isolated from the patient samples and liver cancer cell lines using Trizol according to the manufacturer's instructions (Invitrogen, Carlsbad, CA, USA). The cDNA was synthesized using reverse transcriptase (Invitrogen) and oligo-dT primers, and then subjected to standard PCR using AmpliTaq Gold DNA polymerase (Applied Biosystems, Foster City, CA, USA). Cycling conditions were: $30 \mathrm{sec}$ at $94^{\circ} \mathrm{C} ; 30 \mathrm{sec}$ at $56^{\circ} \mathrm{C}$; and $45 \mathrm{sec}$ at $72^{\circ} \mathrm{C}$ for $32-36$ cycles, followed by a final 7 -min extension step at $72^{\circ} \mathrm{C}$. PCR primers were as follows: $5^{\prime}$-GTACAGGGA CTTCAAGCTACCTC-3' and 5'-TCAGCTGTCAACATGCACAG-3' for TDRD1, 5'-TGTGGT ATCGTGGCAAGGTG-3' and 5'-CAGACATTCCCAACAGGT GTG-3' for TDRD4, 5'-TGGTTCTGTGGAAAGCTCAC-3' and 5'-GCTTCCATCCT TTTCACACTG-3' for TDRD5, 5'-CCAGCC GGACTGAAGAATTG-3' and 5'-GCTTCAATACCGCTGATCAG-3' for CK19, 5'-AACTGCTTGAGCATCAGGATAC-3' and 5'-TGA ATAGTGATGGACCATGGAC-3' for CD133, 5'-TCCCAGCAA CGTGCTTTGAG-3' and 5'-GGACACATCCTCAGGAGAATG-3' for tyrosine aminotransferase (TAT), 5'-ACTTCGCTTCACCTC TGCAC-3' and 5'-CTAACCTCCTCCCCCAACTC-3' for hepatitis B virus $\mathrm{x}$ gene $(\mathrm{HBx})$, and $5^{\prime}$-CTGCACCACCAACTGCTTAG-3' and 5'-TGCTGTAGCCAAATTCGTTG-3' for GAPDH.

The PCR products were analyzed by agarose gel electrophoresis, followed by ethidium bromide staining. Real-time PCR was performed using the SYBR Green PCR kit and ABI 7500 machine (Applied Biosystems) following the manufacturer's instructions. Samples were analyzed in triplicate, and all values are means of triplicate determinations. Relative expression levels of TDRD1 were determined by calculating the (-) $\Delta \Delta \mathrm{CT}$, according to the manufacturer's protocol.

\section{Statistical analysis}

Statistical analysis was performed with SPSS for Windows (version 13.0; Chicago, IL, USA). Fisher's exact test was used to analyze the relationship between pathological parameters and expression levels of TDRD4 and 5.

\section{Results}

\section{Expression of TDRD1, 4, and $5 \mathrm{mRNAs}$ in HCC}

TDRD1, 4, and 5 mRNA expression levels were reported to be detectable in the testis but not in other adult normal tissues of mice (Wang et al. 2001; Smith et al. 2004). The expression of TDRD5 mRNA was evaluated using Northern blot analysis (Smith et al. 2004), which may not be sensitive enough to detect TDRD5 expression in rare cell populations. Therefore, we examined whether this gene and TDRD1 and 4 were expressed in liver tissue as well as other tissue types using RT-PCR. TDRD1, 4, and 5 mRNAs were highly expressed in testis tissue, as expected (Fig. 1a). However, we detected TDRD5 expression in the RNA of the normal colonic mucosa and of the stomach mucosa (Fig. 1a), suggesting that TDRD5 is not a CT antigen in humans. 
a
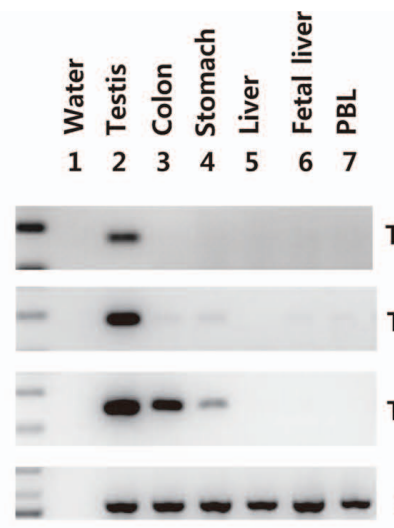

TDRD1

TDRD4

TDRD5

GAPDH

C

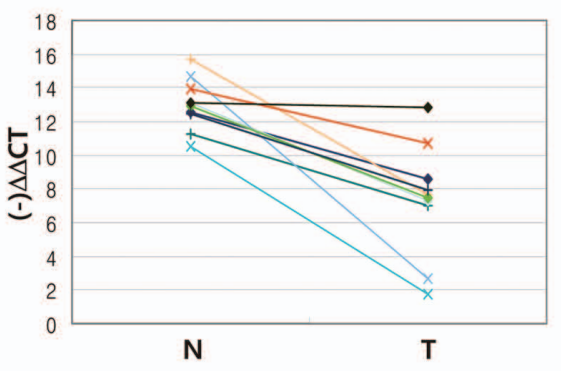

b
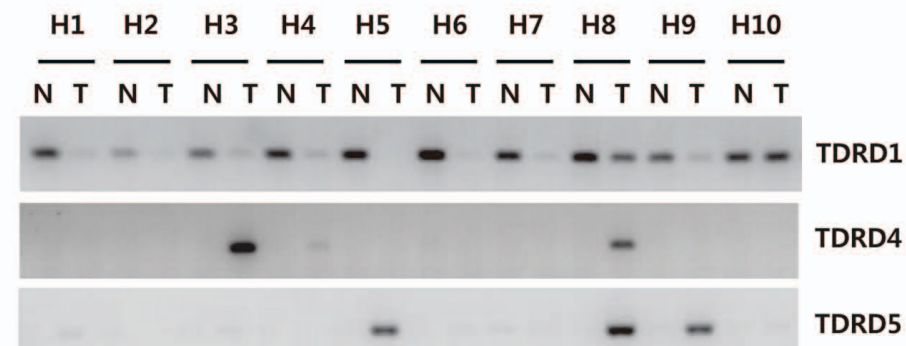

TDRD5

$\mathrm{HBx}$

GAPDH
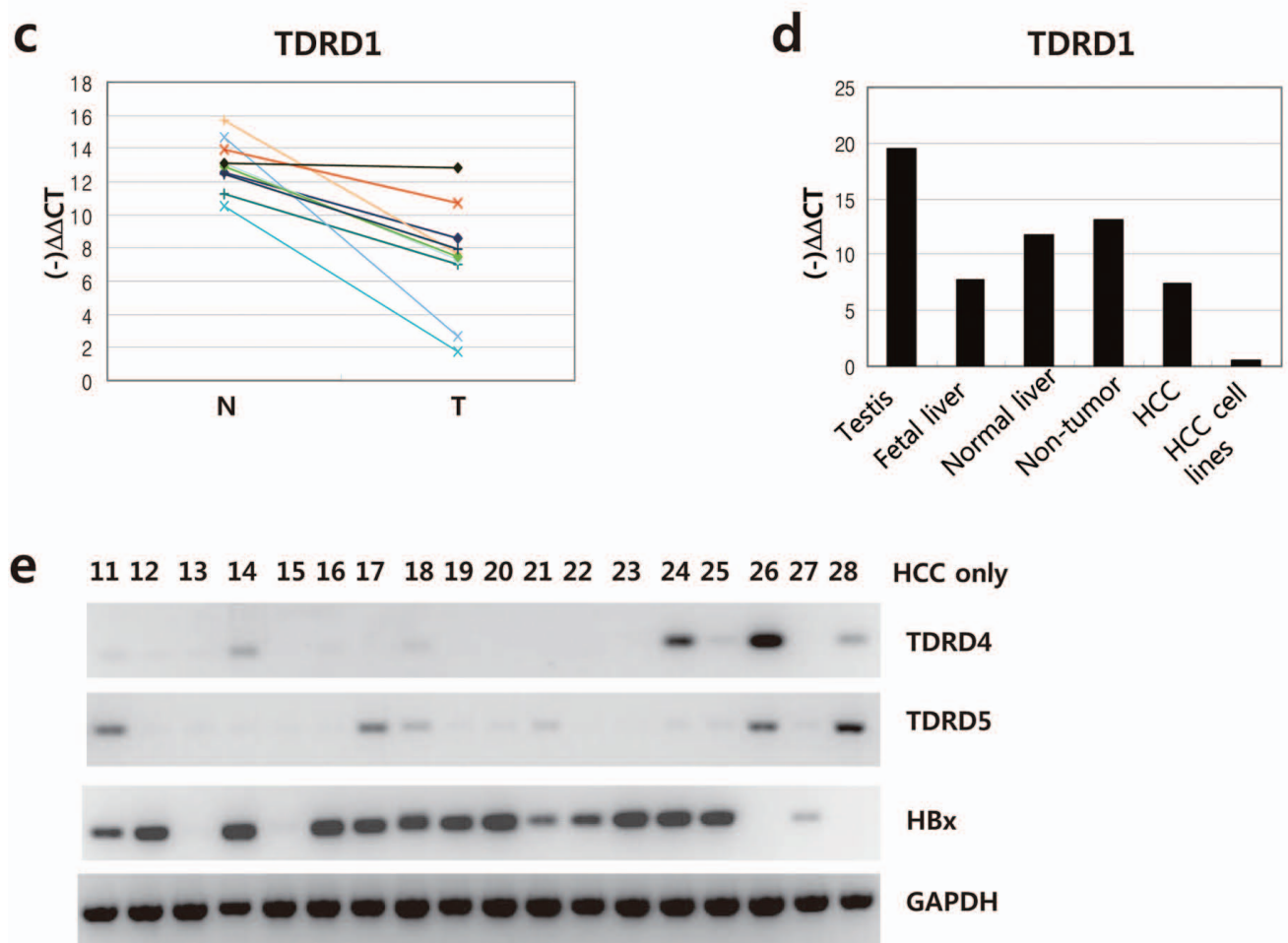

Fig. 1. Expression profiles of TDRD1, 4, and 5 mRNAs in normal tissues and HCCs.

(A) Tissue distribution of TDRD1, 4, and 5 mRNAs was evaluated by RT-PCR. The housekeeping gene, GAPDH, was used as a positive control. TDRD5 is expressed in the normal colonic and the normal gastric mucosa. PBL, peripheral blood lymphocyte. (B) Expression of TDRD1, 4, and 5 mRNAs in matched non-tumorous liver tissue and HCC (N, non-tumor; T, tumor). HBx was used to assess the presence of an HBV infection. TDRD1 was expressed in all nontumorous liver tissues, but only two HCCs (H8-T and H10-T). TDRD4 was expressed in two HCCs (H3-T and H8-T), but not in any non-tumorous tissues. TDRD5 was expressed in three HCCs (H5-T, H8-T and H9-T), but not in any nontumorous tissues. (C) Real-time RT-PCR confirmed higher expression levels of TDRD1 in non-tumorous tissue than HCC. (D) Real-time RT-PCR results of relative TDRD1 expression among the testis, the fetal liver, the normal liver, a non-tumor tissue (H8), a HCC (H8), and a HCC cell line (Hep3B). This result confirmed the unexpected expression of TDRD1 in non-tumorous liver tissue. (E) Expression of TDRD4, TDRD5, and HBx in the remaining 18 HCCs. TDRD4 was expressed in case 24 and 26, and TDRD5 was expressed in case 11, 17, 26, and 28.

We next examined the expression of TDRD1, 4, and 5 mRNAs in HCC specimens using RT-PCR (Fig. 1b). Unexpectedly, TDRD1 was detected in non-tumorous liver tissue, but rarely expressed in HCC samples (Fig. 1b). These results were further confirmed by real-time PCR (Fig. 1c). Since TDRD1 was not detected in the normal liver, but was detected in the non-tumorous liver tissue from HCC patients (Fig. 1a, 1b), we compared the relative expression levels of TDRD1 between them, including fetal liver and HCC cell lines, using real-time PCR. TDRD1 expression 
Table 1. Relationship between clinicopathological characteristics of 28 HCCs and expression of TDRD4 and 5 mRNAs.

\begin{tabular}{|c|c|c|c|c|c|c|}
\hline & $\begin{array}{c}\text { TDRD4 } \\
\text { negative }\end{array}$ & $\begin{array}{l}\text { TDRD4 } \\
\text { positive }\end{array}$ & $p$ value & $\begin{array}{l}\text { TDRD5 } \\
\text { negative }\end{array}$ & $\begin{array}{l}\text { TDRD5 } \\
\text { positive }\end{array}$ & $p$ value \\
\hline \multicolumn{7}{|l|}{ Age (years) } \\
\hline Median & 53 & 57.5 & 0.48 & 52 & 62 & 0.002 \\
\hline \multicolumn{7}{|l|}{ Size } \\
\hline$<5 \mathrm{~cm}$ & 17 & 3 & 0.68 & 14 & 5 & 0.6 \\
\hline$>5 \mathrm{~cm}$ & 7 & 1 & & 7 & 2 & \\
\hline \multicolumn{7}{|l|}{ Edmondson grade } \\
\hline I and II & 12 & 1 & 0.35 & 11 & 3 & 0.8 \\
\hline III and IV & 12 & 3 & & 10 & 4 & \\
\hline \multicolumn{7}{|l|}{ Viral marker } \\
\hline HBV $(+)$ & 21 & 2 & 0.09 & 18 & 2 & 0.005 \\
\hline $\mathrm{HCV}(+)$ & 1 & 0 & & 1 & 0 & \\
\hline $\mathrm{HBV}(-) \mathrm{HCV}(-)$ & 2 & 2 & & 2 & 5 & \\
\hline \multicolumn{7}{|c|}{ Underlying liver disease } \\
\hline Cirrhosis & 12 & 1 & 0.5 & 11 & 2 & 0.64 \\
\hline Chronic hepatitis & 12 & 3 & & 10 & 5 & \\
\hline Total & 24 & 4 & & 21 & 7 & \\
\hline
\end{tabular}

in non-tumorous liver tissues of HCC patients was relatively higher than that in other samples (Fig. 1d). Regarding the expression of TDRD1 in non-tumorous liver tissue, we concluded that TDRD1 is not a CT antigen for HCC.

TDRD4 and 5 were cancer-specifically expressed in a subset of HCCs at frequencies of 14\% (4/28) and 25\% (7/28), respectively (Fig. 1b, 1e). Because TDRD5 was expressed in the normal colonic and gastric mucosa, and TDRD1 was expressed in non-tumorous liver tissue, only TDRD4 shows the potential to be a CT antigen for HCC tumors.

\section{Association between TDRD5 expression and HBx-negative} status

Because the surface antigen of the hepatitis $B$ virus (HBsAg) expression is often negative in cases of occult hepatitis B virus (HBV) infection and initially integrated HBV DNA is often lost from the host genome (Feitelson and Lee 2007), serologic results for HBsAg may not always provide information on the role of oncogenic viral genes, such as HBx. Therefore, we performed RT-PCR to assess $\mathrm{HBx}$ expression in matched non-tumorous and tumor tissues. HBx expression was not exhibited by 5 of the 7 TDRD5-expressing tumors (Fig. 1b, 1e). The association between TDRD5 expression and HBx-negative status was significant ( $p=0.005$; Fisher's exact test, Table 1). In contrast, the expression of TDRD1 or 4 was not associated with HBx status (Fig. 1 b, 1e; Table 1).

\section{TDRD4, TDRD5, and CD133 expression in liver cancers}

We next assessed whether the expression of TDRD4 and 5 were associated with combined HCC-CCs and liver cancer stem cell marker, CD133 (Wu and Wu 2009). Of the eight combined HCC-CCs, five cases expressed CD133.
TDRD4 expression was detected in 2 of the 5 CD133positive combined HCC-CCs (Fig. 2a). All five CCs expressed CD133. Among them, only one case expressed TDRD4 (Fig. 2b). In HCCs, 1 of 3 CD133-positive cases expressed TDRD4 (Fig. 2c). Taken together, there was no significant correlation between TDRD4 and CD133 expression in liver cancers ( $p=0.25$; Fisher's exact test). Likewise, the relationship between CD133 and TDRD5 expression was not observed in the liver cancers (Fig. 2a, 2b). TAT, a hepatocyte differentiation marker, was downregulated in tumor samples expressing TDRD4, implicating an association between poorly differentiated phenotype of HCC and a TDRD4 expression (Fig. 2c).

\section{Discussion}

TDRD1 expression was absent in the normal liver and most HCCs, but was activated in non-tumorous liver tissues of HCCs patients. Thus, it is not a CT antigen or a tumorspecific marker for HCC. We tested the methylation status of the TDRD1 promoter region because promoter hyper- or hypomethylation often underlies the mechanism by which CT antigens are silenced or activated (Cho et al. 2003; Luetkens et al. 2010), TDRD1 was hypomethylated in nontumorous tissues compared to the corresponding HCC tissues (data not shown), consistent with the over-expression of TDRD1 mRNA in non-tumorous tissue. Considering that all 28 HCC patients showed cirrhosis or chronic hepatitis (Table 1), the relationship between TDRD1 mRNA expression and the condition of cirrhosis or chronic hepatitis deserves further evaluation.

TDRD4 was not expressed in non-tumorous liver tissue but expressed only in a subset of liver cancers and in the testis, suggesting that TDRD4 is a CT antigen for liver cancer. To evaluate the potential contribution of TDRD4 to 
a
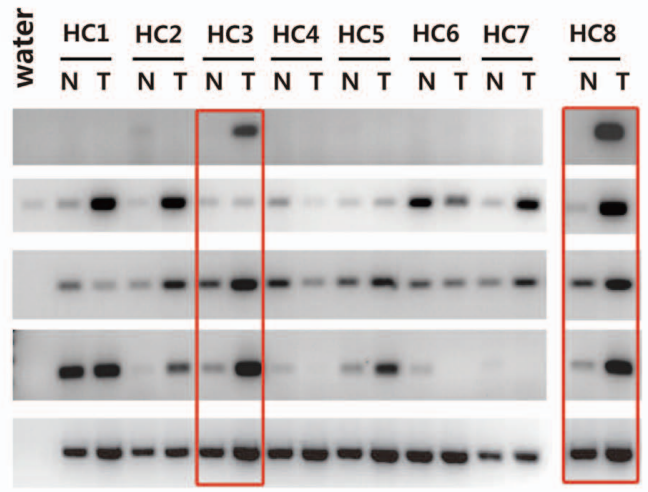

b $\stackrel{\mathrm{d}}{\mathrm{N}}_{\mathrm{J}}^{\mathrm{C}} \frac{\mathrm{C} 1}{\mathrm{~N} T} \frac{\mathrm{C} 2}{\mathrm{~N} T} \frac{\mathrm{C} 3}{\mathrm{~N} T} \frac{\mathrm{C} 4}{\mathrm{~N} T} \frac{\mathrm{C5}}{\mathrm{N} T}$

TDRD4

TDRD5

CK19

CD133

GAPDH

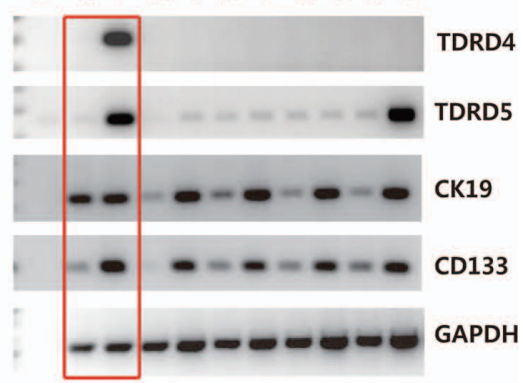

C

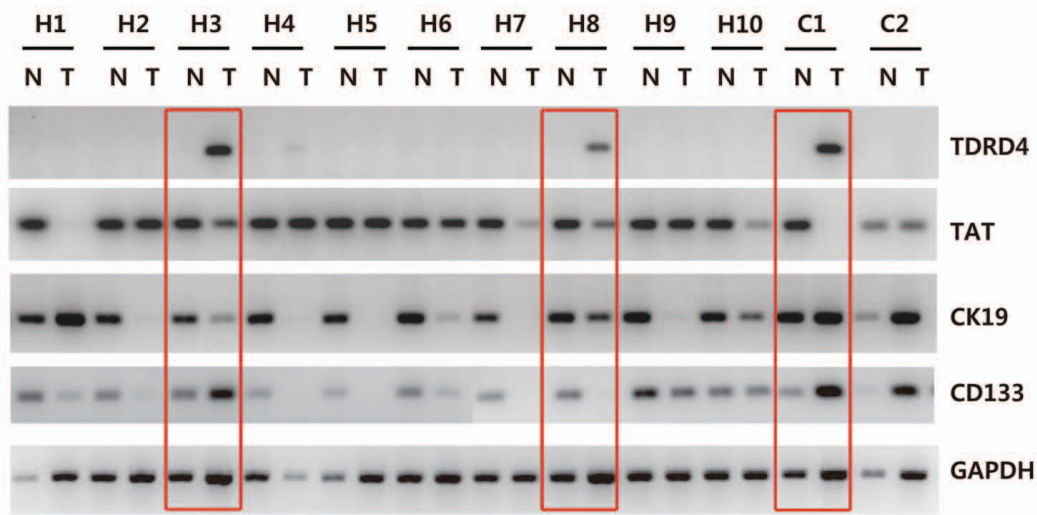

Fig. 2. Expression of TDRD4, TDRD5, CK19, and CD133 mRNAs in the three types of liver cancer.

(A) Eight combined HCC-CCs (HC1 to HC8), (B) five cholangiocarcinomas (C1 to C5), and (C) 10 hepatocellular carcinomas (H1 to H10). RT-PCR was performed for TDRD4 and 5, CK19, and CD133 mRNA expression. GAPDH was used as a loading control. TDRD4 was expressed in 5 of 23 tumors (highlighted with squares): 2 of 8 combined HCC$\mathrm{CCs}$ (HC3 and $\mathrm{HC} 8) ; 1$ of $5 \mathrm{CCs}(\mathrm{C} 1)$; and 2 of $10 \mathrm{HCCs}(\mathrm{H} 3$ and H8). TDRD5 was expressed in 5 of 8 combined HCC-CCs and 2 of 5 CCs, but also in a non-tumorous liver tissue (case HC6). Neither CD133 nor tyrosine aminotransferase (TAT) mRNA expression was associated with TDRD4 or 5 expression.

malignant phenotype, including differentiation and the expression of stem cell marker, we examined mRNA expression patterns of TDRD4 versus CD133 or TAT. CD133 was upregulated in 4 of 5 TDRD4-positive liver cancers and 9 of 18 TDRD4-negative cases. TAT was downregulated in 2 of 2 TDRD4-positive HCC cases and 3 of 8 TDRD4-negative cases (Fig. 2). Although there was no statistically significant correlation between TDRD4 versus CD133 or TAT expression, there was a trend of relative coincidence between the upregulation of CD133 or downregulation of TAT with TDRD4 expression. Further study with a larger series of liver cancers could clarify the relationship between expression of TDRD4 and CD133 or TAT.

Although TDRD5 was revealed not to be a CT antigen, the expression of TDRD5 was associated with the HBxnegative status in HCCs, suggesting that TDRD5 expression is associated with other factors than HBV infection.

In conclusion, we examined the mRNA expression of three TDRDs mRNAs in the three types of liver cancers. Among the TDRDs, TDRD4 is the only candidate CT antigen and might serve as a target for cancer immunotherapy in liver cancer.

\section{Acknowledgments}

This study was supported by a grant from the Korea Health 21 R\&D Project, Ministry of Health and Welfare, Republic of Korea (03-PJ10-PG6-GP01-0002), the Converging Research Center Program through the Ministry of Education, Science and Technology (2010K001115), and a faculty research grant from the Yonsei University College of Medicine for 2010 (6-20100142).

\section{Conflict of Interest}

The authors have no conflict of interest.

\section{References}

Almeida, L.G., Sakabe, N.J., deOliveira, A.R., Silva, M.C., Mundstein, A.S., Cohen, T., Chen, Y.T., Chua, R., Gurung, S., Gnjatic, S., Jungbluth, A.A., Caballero, O.L., Bairoch, A., Kiesler, E., White, S.L., Simpson, A.J., Old, L.J., Camargo, A.A. \& Vasconcelos, A.T. (2009) CTdatabase: a knowledgebase of high-throughput and curated data on cancer-testis antigens. Nucleic Acids Res., 37, D816-819.

Caballero, O.L. \& Chen, Y.T. (2009) Cancer/testis (CT) antigens: 
potential targets for immunotherapy. Cancer Sci., 100, 20142021.

Cho, B., Lee, H., Jeong, S., Bang, Y.J., Lee, H.J., Hwang, K.S., Kim, H.Y., Lee, Y.S., Kang, G.H. \& Jeoung, D.I. (2003) Promoter hypomethylation of a novel cancer/testis antigen gene CAGE is correlated with its aberrant expression and is seen in premalignant stage of gastric carcinoma. Biochem. Biophys. Res. Commun., 307, 52-63.

Chuma, S., Hiyoshi, M., Yamamoto, A., Hosokawa, M., Takamune, K. \& Nakatsuji, N. (2003) Mouse Tudor Repeat-1 (MTR-1) is a novel component of chromatoid bodies/nuages in male germ cells and forms a complex with snRNPs. Mech. Dev., 120, 979-990.

Chuma, S., Hosokawa, M., Kitamura, K., Kasai, S., Fujioka, M., Hiyoshi, M., Takamune, K., Noce, T. \& Nakatsuji, N. (2006) Tdrd1/Mtr-1, a tudor-related gene, is essential for male germcell differentiation and nuage/germinal granule formation in mice. Proc. Natl. Acad. Sci. USA, 103, 15894-15899.

Costa, F.F., Le Blanc, K. \& Brodin, B. (2007) Concise review: cancer/testis antigens, stem cells, and cancer. Stem Cells, 25, 707-711.

Farazi, P.A. \& DePinho, R.A. (2006) Hepatocellular carcinoma pathogenesis: from genes to environment. Nat. Rev. Cancer, 6 , 674-687.

Feitelson, M.A. \& Lee, J. (2007) Hepatitis B virus integration, fragile sites, and hepatocarcinogenesis. Cancer Lett., 252, 157-170.

Gallant, P. (2006) Myc/Max/Mad in invertebrates: the evolution of the Max network. Curr. Top. Microbiol. Immunol., 302, 235253.

Hofmann, O., Caballero, O.L., Stevenson, B.J., Chen, Y.T., Cohen, T., Chua, R., Maher, C.A., Panji, S., Schaefer, U., Kruger, A., Lehvaslaiho, M., Carninci, P., Hayashizaki, Y., Jongeneel, C.V., Simpson, A.J., Old, L.J. \& Hide, W. (2008) Genomewide analysis of cancer/testis gene expression. Proc. Natl. Acad. Sci. USA, 105, 20422-20427.

Koh, K.C., Lee, H., Choi, M.S., Lee, J.H., Paik, S.W., Yoo, B.C., Rhee, J.C., Cho, J.W., Park, C.K. \& Kim, H.J. (2005) Clinicopathologic features and prognosis of combined hepatocellular cholangiocarcinoma. Am. J. Surg., 189, 120-125.

Loriot, A., Boon, T. \& De Smet, C. (2003) Five new human cancer-germline genes identified among 12 genes expressed in spermatogonia. Int. J. Cancer, 105, 371-376.

Luetkens, T., Schafhausen, P., Uhlich, F., Stasche, T., Akbulak, R., Bartels, B.M., Hildebrandt, Y., Gontarewicz, A., Kobold, S.,
Meyer, S., Gordic, M., Bartels, K., Lajmi, N., Cao, Y., Kroger, N., Bokemeyer, C., Brummendorf, T.H. \& Atanackovic, D. (2010) Expression, epigenetic regulation, and humoral immunogenicity of cancer-testis antigens in chronic myeloid leukemia. Leuk. Res., 34, 1647-1655.

Pan, J., Goodheart, M., Chuma, S., Nakatsuji, N., Page, D.C. \& Wang, P.J. (2005) RNF17, a component of the mammalian germ cell nuage, is essential for spermiogenesis. Development, 132, 4029-4039.

Parmigiani, R.B., Bettoni, F., Vibranovski, M.D., Lopes, M.H., Martins, W.K., Cunha, I.W., Soares, F.A., Simpson, A.J., de Souza, S.J. \& Camargo, A.A. (2006) Characterization of a cancer/testis (CT) antigen gene family capable of eliciting humoral response in cancer patients. Proc. Natl. Acad. Sci. USA, 103, 18066-18071.

Simpson, A.J., Caballero, O.L., Jungbluth, A., Chen, Y.T. \& Old, L.J. (2005) Cancer/testis antigens, gametogenesis and cancer. Nat. Rev. Cancer, 5, 615-625.

Smith, J.M., Bowles, J., Wilson, M., Teasdale, R.D. \& Koopman, P. (2004) Expression of the tudor-related gene Tdrd5 during development of the male germline in mice. Gene Expr. Patterns, 4, 701-705.

Theise, N.D., Curado, M.P., Franceschi, S., Hytiroglou, P., Kudo, M., Park, Y.N., Sakamoto, M., Toberson, M. \& Wee, A. (2010) Hepatocellular carcinoma. In WHO classification of tumours of the digestive system, 4th ed., edited by F.T. Bosman, F. Carneiro, R.H. Hruban, \& N.D. Theise. International agency of research on cancer, Lyon, pp. 205-227.

Wang, J., Wang, F. \& Kessinger, A. (2010) Outcome of combined hepatocellular and cholangiocarcinoma of the liver. J. Oncol., Epub 2010.

Wang, P.J., McCarrey, J.R., Yang, F. \& Page, D.C. (2001) An abundance of $\mathrm{X}$-linked genes expressed in spermatogonia. Nat. Genet., 27, 422-426.

Wu, Y. \& Wu, P.Y. (2009) CD133 as a marker for cancer stem cells: progresses and concerns. Stem Cells Dev., 18, 11271134.

Yin, X.Y., Gupta, K., Han, W.P., Levitan, E.S. \& Prochownik, E.V. (1999) Mmip-2, a novel RING finger protein that interacts with mad members of the Myc oncoprotein network. Oncogene, 18, 6621-6634.

Yin, X.Y., Grove, L.E. \& Prochownik, E.V. (2001) Mmip-2/ Rnf-17 enhances c-Myc function and regulates some target genes in common with glucocorticoid hormones. Oncogene, 20, 2908-2917. 